Z Epileptol 2012 $\cdot 25: 241-242$

DOI 10.1007/s10309-012-0283-1

Online publiziert: 18. Oktober 2012

(c) Springer-Verlag 2012

K.E. Brückner ${ }^{1}$ M.T. Lutz ${ }^{2}$

${ }^{1}$ Epilepsiezentrum Hamburg, Evangelisches Krankenhaus Alsterdorf, Hamburg

${ }^{2}$ Fachkrankenhaus für Neurologie, Sächsisches Epilepsiezentrum Radeberg, Radeberg

\title{
Schwerpunktheft Neuropsychologie
}

Auf der Mitgliederversammlung der Deutschen Gesellschaft für Epileptologie (DGfE) in Graz wurde eine neuropsychologische Kommission der DGfE eingerichtet. Diese setzt sich aus in Epilepsiezentren und Epilepsiekliniken tätigen Neuropsychologen zusammen. $\mathrm{Zu}$ den Zielen der Kommission gehören die Weiterentwicklung klinischer Standards in der neuropsychologischen Diagnostik und Therapie bei Epilepsiepatienten sowie die kollegiale Vernetzung und der fachliche Austausch zwischen Neuropsychologen, die sie in regelmäßigen Treffen verfolgen. Die neuropsychologische Kommission der DGfE nahm die Einladung von Herrn Prof. Steinhoff als Mitherausgeber der Zeitschrift für Epileptologie, ein neuropsychologisches Schwerpunktheft mit eigenen Beiträgen zu gestalten, sehr gern an.

In mehreren Arbeitskreissitzungen wurden Empfehlungen zur Standardisierung der prächirurgischen Diagnostik erarbeitet. Diese zentrenübergreifenden Empfehlungen werden im vorliegenden Schwerpunktheft nun erstmalig publiziert. Neben diesem Konsenspapier und 6 Übersichtsbeiträgen wird in einer Originalarbeit die Eignung eines kurzen Screeningverfahrens zur Erkennung depressiver Störungsbilder untersucht.

$\mathrm{Zu}$ den bei Patienten mit Epilepsie am häufigsten beeinträchtigten Funktionsbereichen gehören Gedächtnis und Aufmerksamkeit. In 2 Übersichtsarbeiten werden Ursachen, klinische Relevanz und Möglichkeiten der Therapie dieser kognitiven Funktionsstörungen behandelt. Ein weiterer Beitrag betont die Alltagsrelevanz neuropsychologischer Rehabilitation, wie sie in Bethel seit vielen Jah- ren praktiziert wird. $\mathrm{Zu}$ den Faktoren, die die kognitiven Leistungen beeinträchtigen können, gehört die antikonvulsive Medikation. In einem Literaturüberblick wird die aktuelle Studienlage hinsichtlich der kognitiven Verträglichkeit der neuen Antikonvulsiva dargestellt. Zwei Beiträge befassen sich mit spezifischen Patientengruppen wie Patienten mit neu diagnostizierter Epilepsie und idiopathisch generalisierten Epilepsien sowie deren neuropsychologischen Besonderheiten.

Wir danken allen Autoren dieses Schwerpunkthefts herzlich für die sehr gute Zusammenarbeit und die vielen inhaltlich wertvollen Beiträge und hoffen, einen guten - wenn auch nicht allumfassenden - Überblick über neuropsychologische Themen im Bereich der Epileptologie zu geben.

Katja E. Brückner (Hamburg) und Martin T. Lutz (Radeberg) (im Namen der Mitglieder der neuropsychologischen Kommission der DGfE)

\section{Korrespondenzadresse}

\section{Dr. K.E. Brückner}

Epilepsiezentrum Hamburg, Evangelisches Krankenhaus Alsterdorf Bodelschwinghstr. 24, 22337 Hamburg k.brueckner@eka.alsterdorf.de

\section{Dr. M.T. Lutz}

Fachkrankenhaus für Neurologie, Sächsisches Epilepsiezentrum Radeberg Wachauer Str. 30, 01454 Radeberg m.lutz@kleinwachau.de

Interessenkonflikt. Die korrespondierenden Autoren geben an, dass kein Interessenkonflikt besteht. 


\section{Hier steht eine Anzeige.}

第 Springer 